\title{
Body composition indices of a load-capacity model: gender- and BMl-specific reference curves
}

\author{
Mario Siervo ${ }^{1, *}$, Carla M Prado ${ }^{2}$, Emily Mire $^{3}$, Stephanie Broyles ${ }^{3}$, Jonathan CK Wells ${ }^{4}$, \\ Steven Heymsfield ${ }^{3}$ and Peter T Katzmarzyk ${ }^{3}$ \\ 'Human Nutrition Research Centre, Institute of Cellular Medicine, Newcastle University, Campus of Ageing and \\ Vitality, Newcastle upon Tyne NE4 5PL, UK: ${ }^{2}$ Department of Agricultural, Food and Nutritional Science, Division of \\ Human Nutrition, University of Alberta, Edmonton, Alberta, Canada: ${ }^{3}$ Pennington Biomedical Research Center, \\ Baton Rouge, LA, USA: ${ }^{4}$ Childhood Nutrition Research Centre, UCL Institute of Child Health, London, UK
}

Submitted 23 April 2014: Final revision received 11 July 2014: Accepted 5 August 2014: First published online 15 September 2014

\begin{abstract}
Objective: Fat mass (FM) and fat-free mass (FFM) are frequently measured to define body composition phenotypes. The load-capacity model integrates the effects of both FM and FFM to improve disease-risk prediction. We aimed to derive age-, gender- and BMI-specific reference curves of load-capacity model indices in an adult population ( $\geq 18$ years).

Design: Cross-sectional study. Dual-energy X-ray absorptiometry was used to measure FM, FFM, appendicular skeletal muscle mass (ASM) and truncal fat mass (TrFM). Two metabolic load-capacity indices were calculated: ratio of FM (kg) to FFM $(\mathrm{kg})$ and ratio of TrFM $(\mathrm{kg})$ to ASM $(\mathrm{kg})$. Age-standardised reference curves, stratified by gender and BMI $\left(<25.0 \mathrm{~kg} / \mathrm{m}^{2}, 25 \cdot 0-29 \cdot 9 \mathrm{~kg} / \mathrm{m}^{2}, \geq 30 \cdot 0 \mathrm{~kg} / \mathrm{m}^{2}\right)$, were constructed using an LMS approach. Percentiles of the reference curves were 5 th, 15th, 25th, 50th, 75th, 85th and 95th.

Setting: Secondary analysis of data from the 1999-2004 National Health and Nutrition Examination Survey (NHANES).

Subjects: The population included 6580 females and 6656 males.

Results: The unweighted proportions of obesity in males and females were $25.5 \%$ and $34.7 \%$, respectively. The average values of both FM:FFM and TrFM:ASM were greater in female and obese subjects. Gender and BMI influenced the shape of the association of age with FM:FFM and TrFM:ASM, as a curvilinear relationship was observed in female and obese subjects. Menopause appeared to modify the steepness of the reference curves of both indices.

Conclusions: This is a novel risk-stratification approach integrating the effects of high adiposity and low muscle mass which may be particularly useful to identify cases of sarcopenic obesity and improve disease-risk prediction.
\end{abstract}

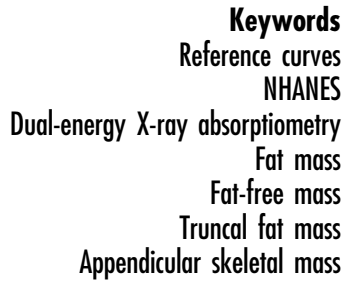

The limitations of anthropometric indices such as BMI and waist circumference (WC) for the prediction of disease risk are known ${ }^{(1,2)}$. WC is generally characterised by an overall greater sensitivity for the prediction of cardiometabolic risk compared with BMI, which is explained by a stronger association with visceral fat ${ }^{(3,4)}$. However, the sensitivity of both BMI and WC for the prediction of cardiometabolic disorders, such as type 2 diabetes and CVD, is modest (diabetes: $\sim 0.67$ for BMI and $\sim 0.72$ for WC; CVD: $~ 0.61$ for BMI and $\sim 0.67$ for $\mathrm{WC})^{(5)}$. The major limitation of these indices is related to their inability to discriminate between fat mass (FM) and fat-free mass (FFM) and hence capture the physiological role that these two components may have on disease risk at different stages of life ${ }^{(6-8)}$. Body composition assessment is therefore important to differentiate the contribution of adiposity and lean mass and improve risk prediction ${ }^{(9)}$. However, body composition research has predominantly examined the association of individual body components with disease risk whereas the additive, interactive effects of adiposity and lean mass have been largely under-investigated ${ }^{(10)}$.

Classification approaches to sarcopenic obesity (SO) have attempted to integrate the within-individual effects derived from the simultaneous presence of excess adiposity (obesity) and low skeletal muscle mass (sarcopenia) ${ }^{(11,12)}$ since they are both independently associated with an increased risk for cardiometabolic disorders and physical disability ${ }^{(13,14)}$. However, the studies investigating the role of SO as a health 
risk factor have mainly been cross-sectional and conducted in older individuals ${ }^{(10)}$. Additionally, the classification of SO has been predominantly based on the application of genderspecific cut-offs, derived from reference groups of young normal-weight subjects, for the identification of sarcopenia and established cut-offs of adiposity indices (i.e. BMI, WC, FM\%) for the assessment of obesity ${ }^{(15)}$. These classifications have ignored the direct association between body mass and body components and applied the same cut-off values for sarcopenia to normal-weight, overweight and obese individuals without taking into consideration the physiological increase in FFM with body mass, which may have affected the specificity of the SO diagnosis (i.e. misclassification of non-sarcopenic obese subjects) ${ }^{(16)}$.

The heterogeneity of SO diagnosis has been further increased by the application of different adiposity indices (BMI, WC, FM\%, fat mass index (FMI), visceral fat area), cutoffs (quartiles, quintiles, $-2 \mathrm{sD}$, predefined cut-offs) and body composition methods (anthropometry, dual-energy X-ray absorptiometry (DXA), computed tomography (CT) scanning, bioelectrical impedance analysis (BIA), airdisplacement phlethysmography $(\mathrm{ADP}))^{(10)}$. Studies have therefore overlooked the dynamic, inter-dependent relationship between FM and lean body mass and their close association with ageing and gender ${ }^{(11,17)}$. These two factors are critical for the identification of SO cases as a decrease in lean tissue density, determined by a higher proportion of connective tissue in the skeletal muscle, has been observed in older and obese subjects ${ }^{(18,19)}$. In addition, the relationship between skeletal muscle and adipose tissue changes with increasing adiposity and it is modified by gender and assessment of regional body composition ${ }^{(16)}$.

A new conceptual approach to chronic disease risk has focused on the contribution of two contrasting traits: (i) 'metabolic capacity', which refers to the organs and tissues that maintain homeostasis; and (ii) 'metabolic load', which derives from other body components or from behaviours (dietary intake, sedentary behaviour) that collectively challenge the maintenance of homeostasis ${ }^{(20,21)}$. The original model focused on the fact that many homeostatic organs are strongly influenced by fetal growth, indicating that metabolic capacity is powerfully shaped by developmental experience. Metabolic load is assumed to emerge through the life course. In adults, high levels of adiposity, sedentary behaviour and lipogenic diet all exacerbate metabolic load ${ }^{(22,23)}$.

This approach can be adapted to SO by treating lean mass, in particular skeletal muscle mass, as a dimension of metabolic capacity especially relevant for physical activity, with further implications for fuel utilisation. Each of high metabolic load (obesity) and low metabolic capacity (low muscle mass) reduces the capacity for physical activity and challenges glucose homeostasis, thereby elevating chronic disease risk.

We have explored the applicability of this model to the diagnosis of SO and assigned 'metabolic load' and 'metabolic capacity' to FM and FFM components, respectively ${ }^{(10)}$. These two components are easily obtained from body composition measurements performed by DXA, BIA or $\mathrm{ADP}^{(24)}$. The application of DXA allows the calculation of a load-capacity model including truncal fat mass (TrFM) and appendicular skeletal muscle mass (ASM) ${ }^{(25)}$ and this index may represent a better predictor of disease risk as it is more closely linked with pathogenetic models of metabolic syndrome and atherosclerosis $^{(26)}$.

The load-capacity model can therefore be translated into a specific diagnostic paradigm to define more sensitive body composition phenotypes for disease-risk prediction. We aimed to use the DXA adult ( $\geq 18$ years) data set from the 1999-2004 National Health and Nutrition Examination Survey (NHANES) to develop age-, gender- and BMIspecific reference curves for two indices (FM:FFM and TrFM: ASM) of a load-capacity model, which can be applied to stratify individuals' health risk according to defined body composition phenotypes.

\section{Methods}

\section{Study sample}

Whole-body DXA measurements on adults ( $\geq 18$ years) from 1999-2004 NHANES were used. A complex multistage probability sampling method was used, which included oversampling of African Americans, Mexican Americans, low-income White and older individuals ( $\geq 60$ years). Household interviews and detailed physical examinations were performed in mobile examination centres. Approval for NHANES 1999-2004 was obtained from the National Center for Health Statistics Institutional Review Board ${ }^{(27)}$.

\section{Subjects}

Reference curves were developed for the total adult population and stratified by gender (males, females) and BMI. BMI categories were: underweight and normal weight ( $\leq 24.9 \mathrm{~kg} / \mathrm{m}^{2}$; UW-NW), overweight (25.0-29.9 kg/m² OW) and obesity ( $\left.\geq 30.0 \mathrm{~kg} / \mathrm{m}^{2} ; \mathrm{OB}\right)$. The underweight and normal weight categories were combined, as were the obese and morbidly obese categories, because of the limited sample size of these groups and they were kept in the final analyses to maintain the representativeness of the reference curves. We have not stratified our population by ethnicity. Prior work on the same cohort has shown that FMI cut-offs that gave the same prevalences as BMI thresholds were similar among the three ethnic groups regardless of the wide variability in BMI prevalence ${ }^{(28)}$. Exclusion criteria were: (i) positive pregnancy test and self-reported pregnancy (women); (ii) taking tests with radiographic contrast material or having participated in nuclear medicine studies in the past $3 \mathrm{~d}$; (iii) self-reported weight (>300 lb $(\sim 136 \mathrm{~kg})$ ) or height $(>6.5 \mathrm{ft}(\sim 1.98 \mathrm{~m})$ ) above the DXA table limit ${ }^{(29)}$; and (iv) missing BMI or DXA measurements of FM. 
(a)

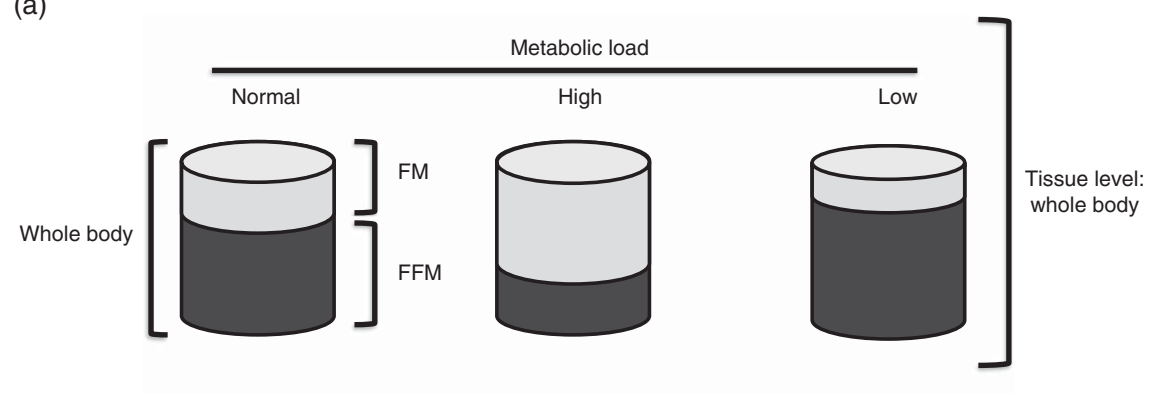

(b)

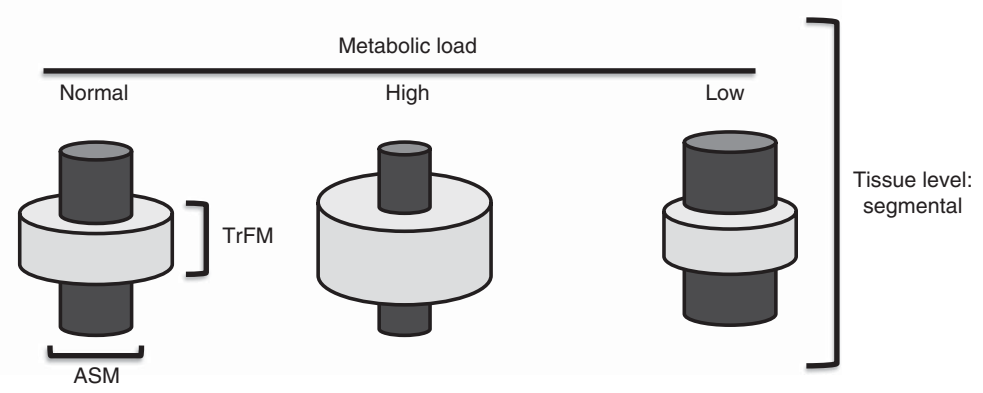

Fig. 1 Whole-body and segmental body composition models of metabolic load. The whole-body model is based on the ratio of fat mass (FM) to fat-free mass (FFM), model a; whereas the segmental model is based on the relationship between truncal fat mass (TrFM) and appendicular skeletal muscle mass (ASM), model b. The metabolic load has been divided in normal, high and low according to the relative contributions of the components. The fundamental concept of the two indices is that the metabolic load in an individual is not given by a specific amount of the two individual components but by their contribution relative to one another. Adapted from Wells ${ }^{(20,21)}$

\section{Body composition measurements}

\section{Anthropometry}

Height was measured using a stadiometer after deep inhalation. Weight was measured using an electronic digital scale calibrated in kilograms. BMI was calculated as weight divided by height squared $\left(\mathrm{kg} / \mathrm{m}^{2}\right)$.

\section{Body composition measurement}

The whole-body DXA exams in NHANES were acquired on a QDR 4500A fan beam densitometer (Hologic, Inc., Bedford, MA, USA) following the manufacturer's recommended acquisition procedures. Hologic DOS software version 8.26: a3* was used to acquire all scans; scanning was done in the fast mode. All subjects changed into paper gowns and were asked to remove all jewellery and other personal effects that could interfere with the DXA exam. The DXA exams were reviewed and analysed by the University of California, San Francisco Department of Radiology Bone Density Group using industry standard techniques. Analysis of all exams was performed using Hologic Discovery software version $12 \cdot 1$ in its default configuration. DXA exams that contained artifacts (i.e. prosthetic devices, pacemakers) that could affect the accuracy of the DXA scan or if data for any region were missing (regional data were summed to equal total values) were coded as invalid and set to missing in the data set. The bone mineral density and content results were calibrated by the DXA manufacturer and maintained by an internal reference system that periodically measures bone and soft tissue equivalent reference standards during the measurement of patients. Details of the calibration protocols have been reported elsewhere ${ }^{(28,29)}$.

\section{Body composition components}

Whole-body DXA measurements of FM (g) and lean mass including bone mineral content (FFM, g) along with demographic information for each subject were used in the analyses. Measurements were also available for segmental regions, such as head, arms, legs, trunk, pelvic regions, sub-total whole body (excluding only the head) and whole body. Leg and arm lean tissue mass represent the sum of both right and left extremities, respectively. ASM was determined by combining the lean tissue mass of the regions of arms and legs, excluding all other body regions from analysis. TrFM was calculated as the difference between total FM and appendicular FM.

\section{Load-capacity model}

The ratios FM:FFM and TrFM:ASM were calculated as measures of the load-capacity model. The model assigns a negative effect on physiological function to FM and TrFM and a positive effect to FFM and ASM. A conceptual description of the model has been provided in Fig. 1. Reference curves were derived for the indices and abnormal body composition phenotypes were identified using conventional cut-offs and classified as moderate if greater than or equal to the 85 th percentile and severe if greater than or equal to the 95th percentile. 


\section{Statistical methods}

Analyses were conducted utilising the DXA data sets released by NHANES on the Centers for Disease Control and Prevention website (http://www.cdc.gov/nchs/about/ major/nhanes/dxx/dxa.htm). Age was entered into the models as the independent variable. Missing data were not completely random and therefore were imputed at the National Center for Health Statistics ${ }^{(30)}$.

A curve-fitting procedure called LMS (ImsChartMaker ProVersion 2.3) was used to generate the reference curves accounting for skewness of the variables ${ }^{(31)}$. The underlying reference data are normalised by dividing the independent measure (e.g. age) into groups and then applying a power transformation to eliminate the skewness of the dependent variable. A smooth curve is fitted to the normalising power transformation for each age group, generating an optimum 'L' (power) curve that normalises the dependent measure over the entire age range. The procedure also fits median ('M') and coefficient of variation ('S') curves, and these three curves (L, M and S) fully describe the reference data. The gender- and BMI-specific percentiles for FM:FFM and TrFM: ASM were 5th, 15th, 25th, 50th, 75th, 85th and 95th. The $Z$-scores for each individual value of the two body composition indices can also be calculated using the equation:

$$
Z=\frac{M\left[(X / M)^{L}-1\right]}{L \sigma},
$$

where $X$ is the body composition ratio (e.g. FM:FFM, TrFM: $\mathrm{ASM}), L$ is the power transformation, $M$ is the median value and $\sigma(S \times M)$ is the population standard deviation. During the curve-fitting process, the weighted observations from the NHANES data sets were fitted by selecting more parsimonious models to avoid over-fitting the curves. The global fit of the smoothed curves was assessed by $Q$ statistics, which were optimum between -2 and 2 but considered acceptable up to a value of 4 for the L, M and $\mathrm{S}$ curves and if the fitted curve was reasonably smooth and plausible for the data being fitted ${ }^{(31)}$. The LMS curve-fitting procedure adjusts for skewness so that the percentile values and $Z$-scores generated by the LMS values are robust when the data are not normally distributed ${ }^{(31)}$.

Reference curves for FM:FFM and TrFM:ASM were derived for the whole data set and then applied after stratification of the sample by gender and BMI.

\section{Results}

The number of subjects for each gender, age and BMI stratum is provided in Table S1 in the online supplementary material. The total number of subjects included in the analyses was 13236 (6656 males, 6580 females) and the unweighted proportions of UN-NW, OW and OB were $35.2 \%, 34.8 \%$ and $30.0 \%$, respectively. The proportion of OB females was higher than males (34.7\% v. $25.5 \%)$ and a similar difference was observed in young and middle-aged subjects (18-59 years: $34.2 \% v .24 \cdot 5 \%$ ) and older subjects ( $\geq 60$ years: $35 \cdot 6 \% v \cdot 27 \cdot 6 \%$ ).

Reference curves were developed for males and females in the whole sample and for each BMI group.

\section{FM:FFM index}

The association between age and FM:FFM was modified by gender and BMI. In females, the index increased progressively with age, it increased in middle-aged women and started to decline after 70 years of age. The stratification by BMI indicated specific patterns showing a direct association of the index with age in all BMI groups, a greater increase in the index in UN-NW young individuals and a greater decline in OW and OB individuals (Fig. 2(a)-(d)). In men, the relationship between age and FM:FFM was overall linear. After stratification by BMI, the linearity was still observed in UN-NW subjects whereas a U-shaped curvilinear association was observed in the OB group (Fig. 3(a)-(d)). The specific FM:FFM percentile values for the male and female groups are reported in the online supplementary material, Tables S2-S9. In addition to the 50th percentile ' $\mathrm{M}$ ', each table also provides the ' $\mathrm{L}$ ' and ' $\mathrm{S}$ ' values, which can be used to calculate $Z$-scores for individuals. The FM:FFM index was greater in females as the 50th percentile curve (median) for UN-NW females showed similar values to that for OB males (Fig. 6(a)).

\section{TrFM:ASM index}

The shape of the association between age and TrFM:ASM was influenced by gender and BMI. Females showed again a progressive increase with age which started to decline after 70 years of age. However, the increase in young age was mostly accounted by the UN-NW and OW groups whereas a marked decline was mostly observed in the $\mathrm{OB}$ female group (Fig. 4(a)-(d)). UN-NW and OW males again showed a linear association between age and TrFM:ASM index, whereas the relationship was characterised by a sigmoidal shape in the OB group (Fig. 5(a)-(d)). The specific TrFM:ASM percentile values for the male and female groups are reported in the online supplementary material, Tables S10-S17. In addition to the 50th percentile ' $\mathrm{M}$ ', each table also provides the ' $\mathrm{L}$ ' and ' $\mathrm{S}$ ' values, which can be used to calculate $Z$-scores for individuals. The TrFM:ASM index was greater in females as the 50th percentile curve (median) of the OB male group was marginally higher than that of UN-NW females (Fig. 6(b)).

\section{Discussion}

Population-representative reference curves for indicators of the load-capacity model (FM:FFM and TrFM:ASM) in adult male and female subjects using NHANES DXA body composition data were derived. We believe that these percentiles and $Z$-scores may improve the assessment of 
(a)

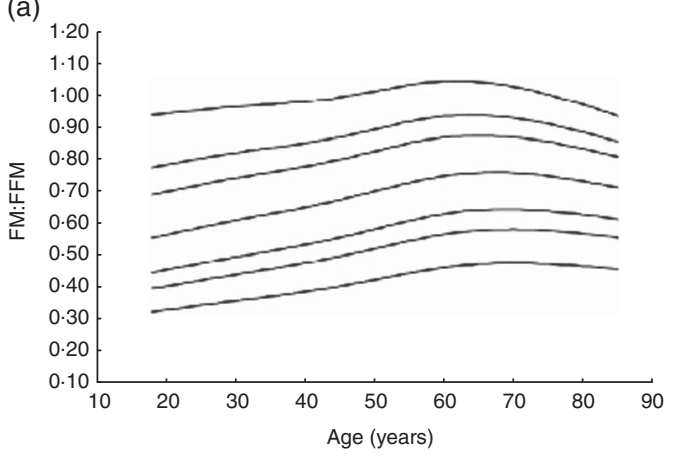

(c)

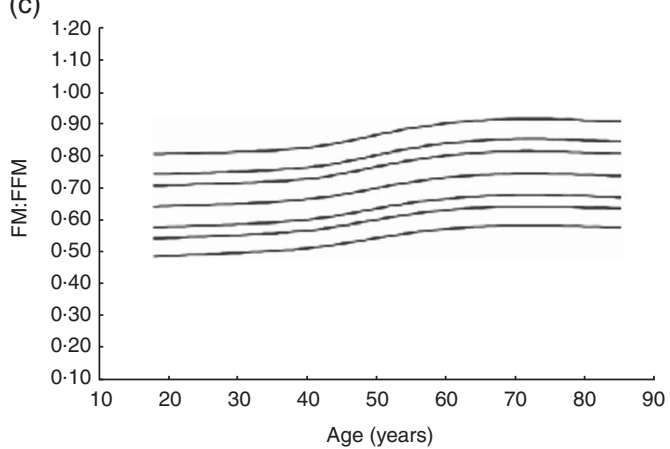

(b)

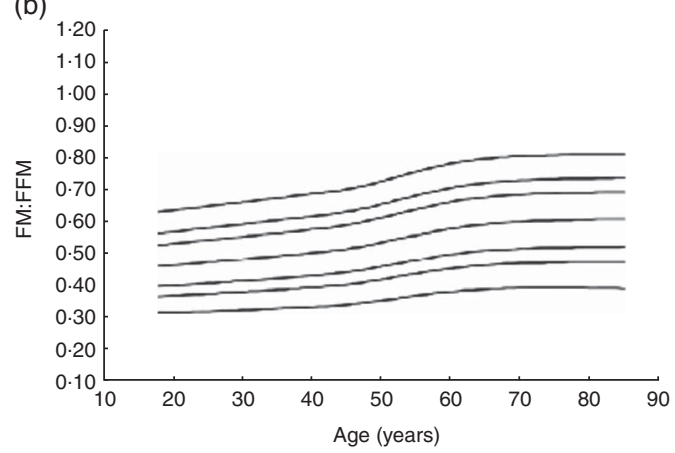

(d)

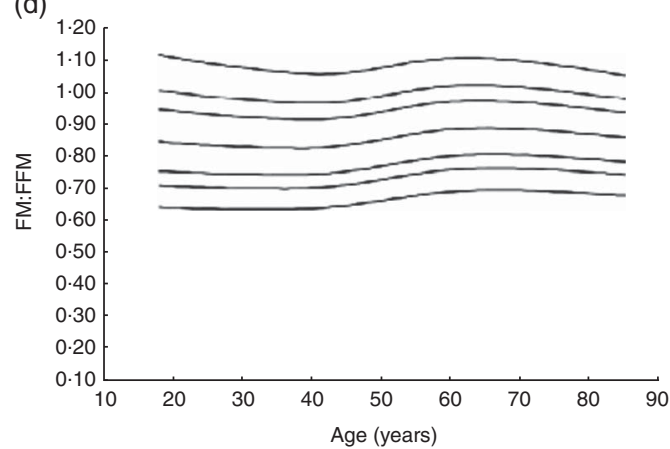

Fig. 2 Reference curves of the ratio of fat mass (FM) to fat-free mass (FFM) for women in the whole population (a) and stratified by BMI as underweight-normal weight (b), overweight (c) and obese (d). Percentiles shown are 5th, 15th, 25th, 50th, 75th, 85th and 95th. Secondary analysis of data from the 1999-2004 National Health and Nutrition Examination Survey (NHANES), $n 6580$

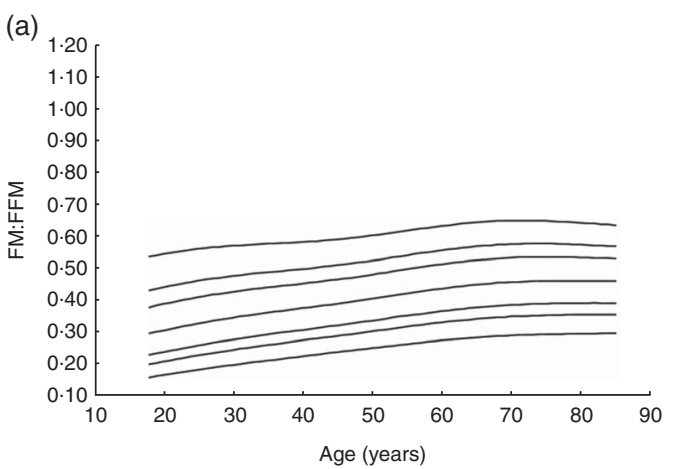

(c)

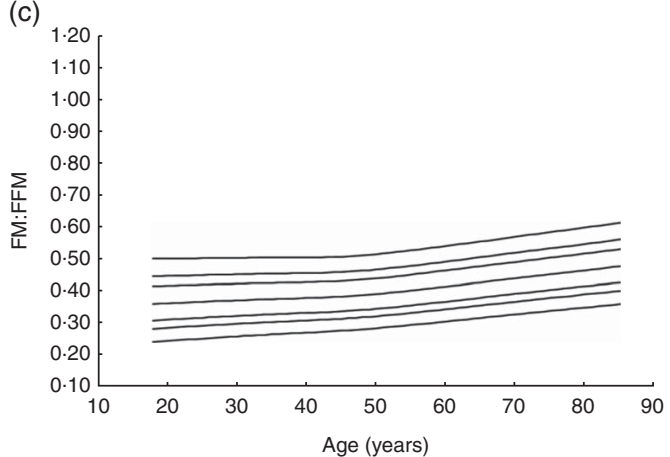

(b)

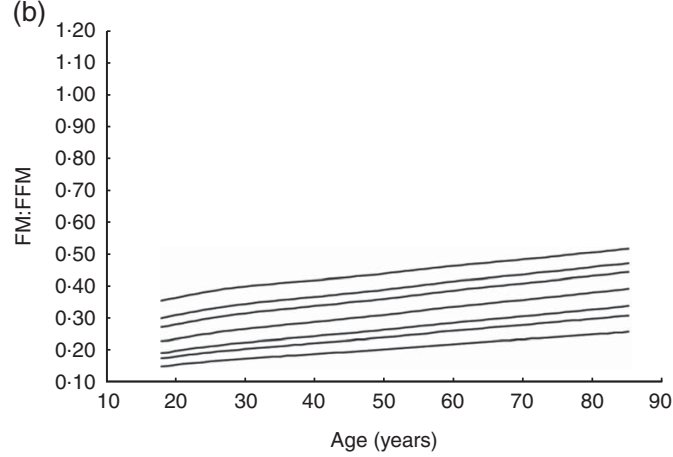

(d)

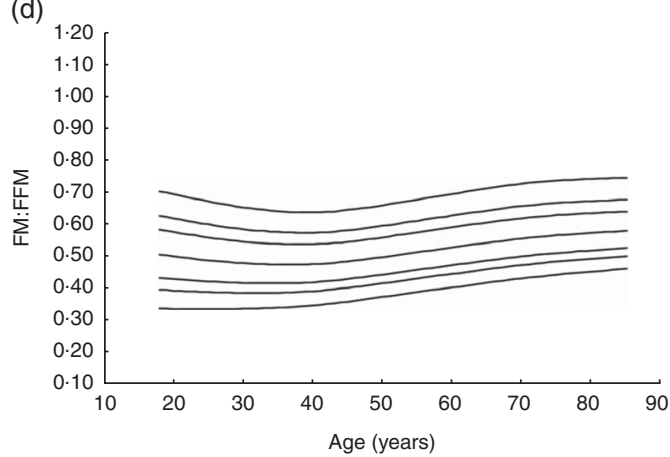

Fig. 3 Reference curves of the ratio of fat mass (FM) to fat-free mass (FFM) for men in the whole population (a) and stratified by BMI as underweight-normal weight (b), overweight (c) and obese (d). Percentiles shown are 5th, 15th, 25th, 50th, 75th, 85th and 95th. Secondary analysis of data from the 1999-2004 National Health and Nutrition Examination Survey (NHANES), n 6656 
(a)

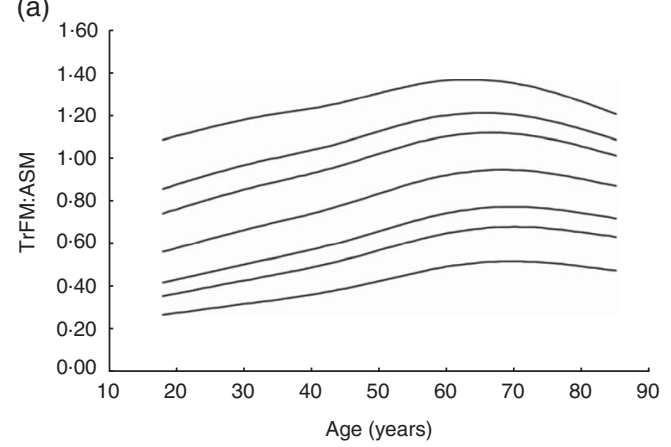

(c)

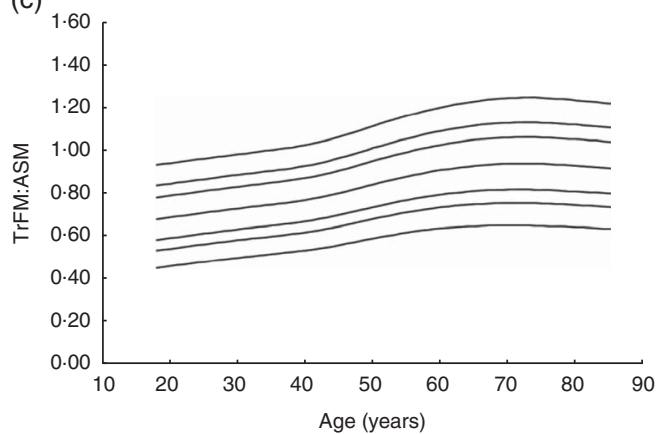

(b)

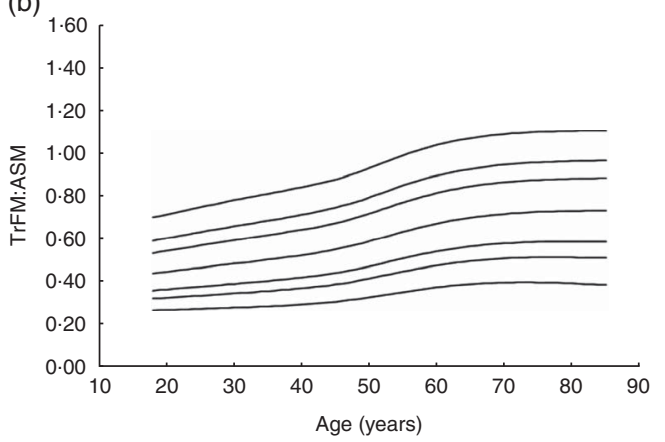

(d)

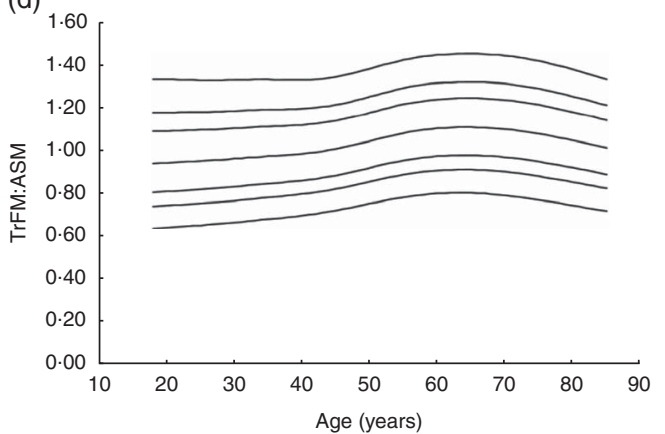

Fig. 4 Reference curves of the ratio of truncal fat mass (TrFM) to appendicular skeletal muscle mass (ASM) for women in the whole population (a) and stratified by BMI as underweight-normal weight (b), overweight (c) and obese (d). Percentiles shown are 5th, 15th, 25th, 50th, 75th, 85th and 95th. Secondary analysis of data from the 1999-2004 National Health and Nutrition Examination Survey (NHANES), $n 6580$

(a)

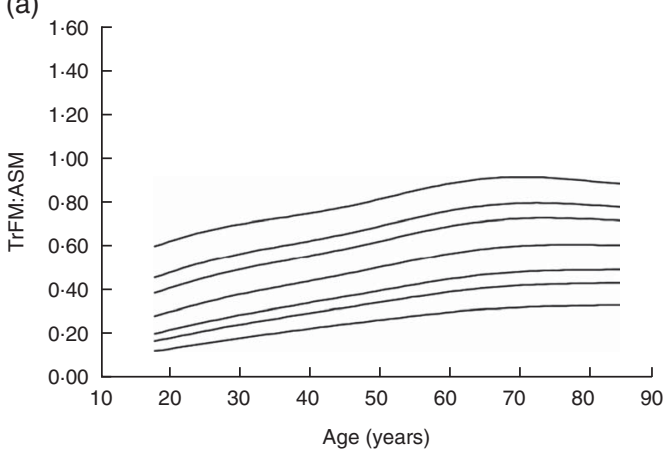

(c)

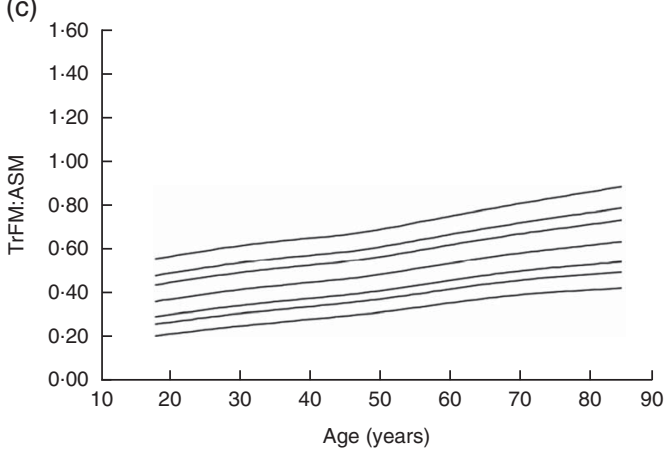

(b)

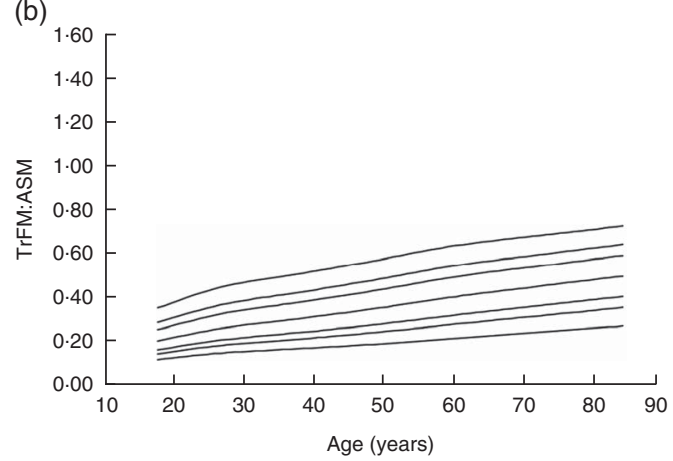

(d)

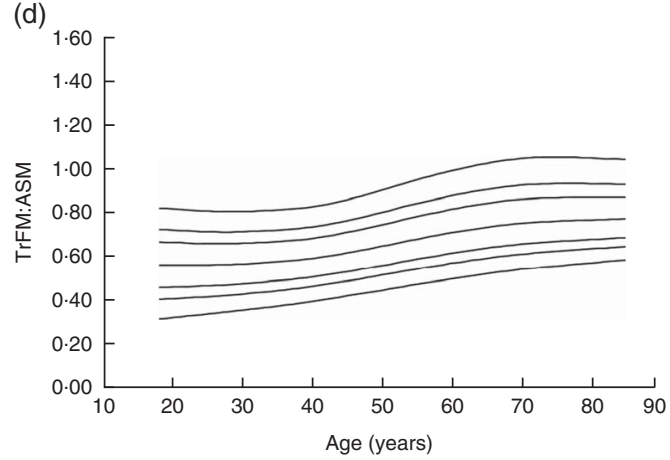

Fig. 5 Reference curves of the ratio of truncal fat mass (TrFM) to appendicular skeletal muscle mass (ASM) for men in the whole population (a) and stratified by BMI as underweight-normal weight (b), overweight (c) and obese (d). Percentiles shown are 5th, 15th, 25th, 50th, 75th, 85th and 95th. Secondary analysis of data from the 1999-2004 National Health and Nutrition Examination Survey (NHANES), $n 6656$ 

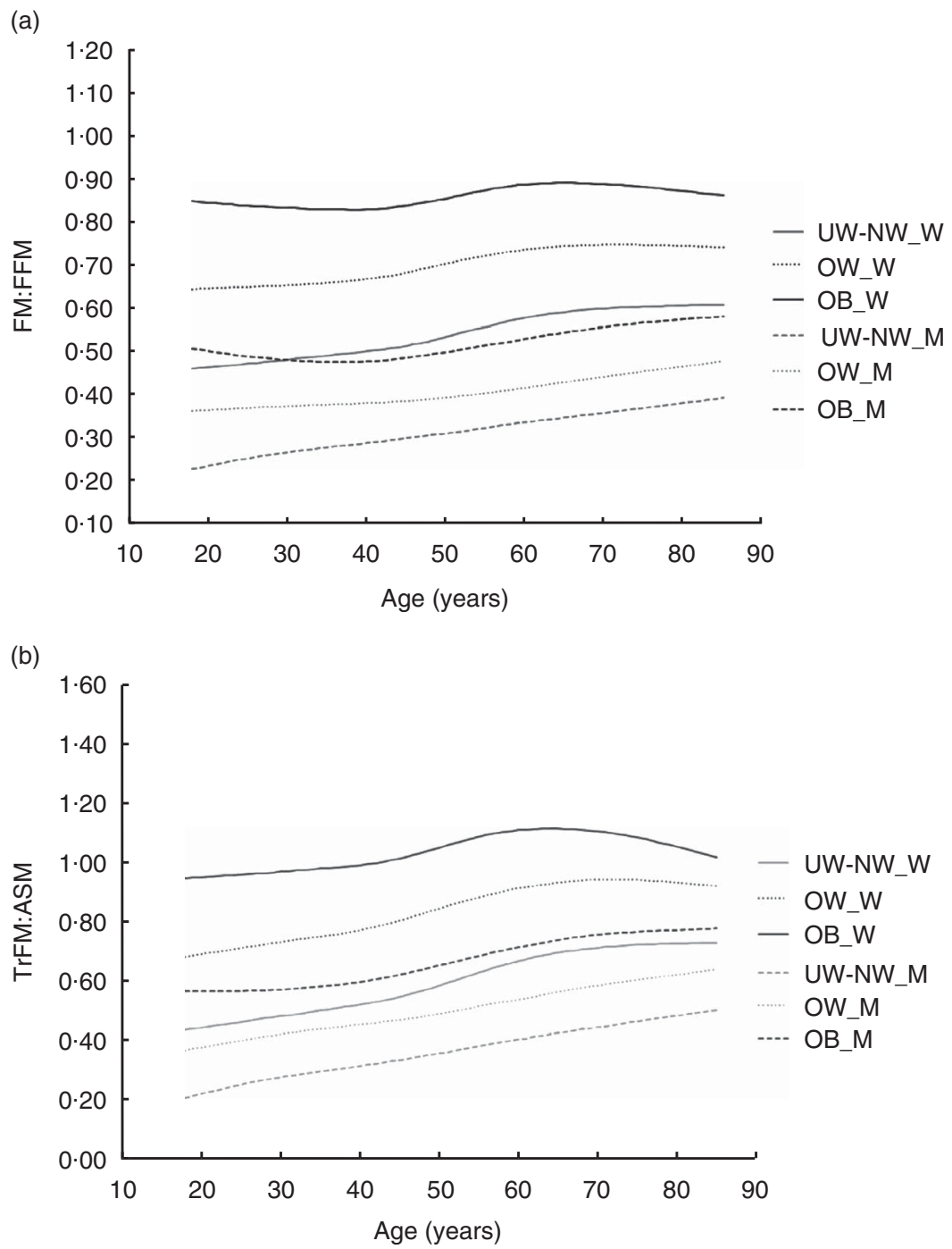

Fig. 6 Median (50th percentile) curves of the fat mass (FM) to fat-free mass (FFM) ratio (a) and the truncal fat mass (TrFM) to appendicular skeletal muscle mass (ASM) ratio (b) for men (M; $n$ 6656) and women (W; $n$ 6580) stratified by BMl as underweight-normal weight (UN-NW), overweight (OW) and obese (OB). Secondary analysis of data from the 1999-2004 National Health and Nutrition Examination Survey (NHANES)

body composition by taking into account age-, genderand BMI-specific influences on body components.

There is a lack of consensus on the criteria for the diagnosis of $\mathrm{SO}$ and several classifications with variable predictive values have been proposed ${ }^{(10,15,32)}$. Most importantly, these classifications have been 'ageing-centred' as they have focused mostly on the diagnosis of $\mathrm{SO}$ in older subjects and failed to recognise the co-occurrence of low muscle mass and high adiposity in young and middle-aged individuals ${ }^{(10)}$. These methodological limitations were demonstrated in a recent study conducted on the same NHANES DXA data set used in our analyses. Batsis et al. conducted a systematic review of the most frequently applied definitions for the diagnosis of $\mathrm{SO}$ and found that the prevalence of $\mathrm{SO}$ ranged from $4.4 \%$ to $84.0 \%$ in men and from $3.6 \%$ to $94.0 \%$ in women ${ }^{(15)}$. As expected, the prevalence of SO increased with age since the cut-offs for sarcopenia were all derived from healthy, young, normal-weight samples. The study did not evaluate the predictive accuracy for disease risk of the different SO definitions and it is reasonable to assume that the same variability may have been observed in the SO risk estimates for cardiometabolic and musculoskeletal disorders. The authors concluded with a final remark on the need for harmonisation of diagnostic criteria for SO. The present analysis aims to resolve this research gap by proposing a novel approach to the diagnosis of body composition phenotypes which incorporates a stratified risk approach to identify subjects with low muscle mass and high adiposity.

The advantages of the proposed approach are several. The representativeness of the population, the large sample size and life-course assessment of body composition $(\geq 18$ years) allowed the development of gender- and BMIspecific reference curves. The proposed approach also 
accounts for the effects of critical confounding factors (age, gender and body mass) influencing the discrimination of body composition phenotypes. DXA has been considered as the method of choice for the diagnosis of SO because it derives a three-component model (adipose tissue, lean body mass and body mineral mass) and provides segmental information on the content of adipose tissue and lean body mass in appendicular and truncal body segments. The application of age-specific cut-offs may also minimise diagnostic bias related to the effects of ageing on the validity of DXA body composition measurements (change in hydration and bone mineral mass). The availability of the DXA segmental measurements provided the opportunity to develop a model based on the close association of abdominal fat accumulation (TrFM) with metabolic impairment and ASM with oxidative functions and metabolic flexibility ${ }^{(8)}$. This model implies the availability of DXA measurements, whereas the FM:FFM model may be characterised by a larger applicability since FM and FFM are commonly measured by several body composition methods (e.g. ADP, BIA, isotope dilution). The agreement between DXA and ADP is moderate as ADP tends to underestimate FFM and the measurement bias is influenced by gender and $\operatorname{adiposity}^{(33-35)}$. Therefore, the application of the DXA cutoffs derived in the present analysis to measurements obtained from other body composition methods needs to be carefully considered and results interpreted with caution.

The load-capacity model is operationalising the assessment of body composition phenotypes by integrating the physiological effects of both adiposity and lean body mass components within the same individual. Risk will therefore derive from the relative contribution of each component to physiological functions, which takes into consideration hormonal and age-related body composition changes. Grijalva-Eternod et al. have evaluated the association of the load-capacity model with blood pressure in the Avon Longitudinal Study of Parents and Children (ALSPAC) cohort and found an increased risk of high blood pressure in subjects with an increased metabolic load ${ }^{(36)}$. Sternfeld et al. reported for the first time, in 1655 older subjects, a direct association of higher ratio of lean mass to FM measured by BIA with faster walking speed and less functional limitation ${ }^{(37)}$. The ratio of visceral fat to thigh muscle area, measured by CT scan, was significantly associated with an increased risk of metabolic syndrome in adult Koreans ${ }^{(38)}$. A DXA-derived adiposity to lean mass ratio predicted a 4 -year increased risk of physical disability in Chinese older subjects and the study reported a U-shaped association of the ratio in men and a linear association in women ${ }^{(39)}$.

The cross-sectional design of the present study did not allow the evaluation of longitudinal changes in body composition. Body composition was measured by DXA and therefore assumptions related to hydration and tissue density have to be taken into account in the interpretation of the results and application of the cut-offs obtained from the analysis. Multiple imputation methods were used to adjust for missing DXA data and a discussion on the validity of the assumptions about the missing DXA data has been provided previously ${ }^{(30)}$. The limited number of UW subjects did not allow the development of reference curves for this group and therefore UW subjects were included in the NW group to preserve the representativeness of the reference curves. As already mentioned in the Methods section, analyses were not stratified by ethnicity after taking into consideration previous results conducted on the same data set indicating similar sensitivity of FMI cut-offs in different ethnic groups ${ }^{(21)}$. Additionally, the stratification by ethnicity (three groups) and BMI (three groups) may have resulted in the development of eighteen (nine for men and nine for women) sets of reference curves which could have greatly impacted on the applicability of the reference curves as well as on the reliability of the estimates. The exclusion of very tall $(>6.5 \mathrm{ft}(\sim 1.98 \mathrm{~m}))$ and morbidly obese individuals ( $>300 \mathrm{lb}$ $(\sim 136 \mathrm{~kg}))$, due to the limited technical capability of the DXA scanner, may limit the application of the proposed body composition reference curves to individuals exceeding these phenotypic characteristics. Furthermore, the NHANES DXA data set is instrument specific (Hologic) and employed adjustments proposed by Schoeller et al. ${ }^{(40)}$, which limits generalisation to a different manufacturer. We urge DXA manufacturers to coordinate DXA calibration (i.e. universal calibration) to allow comparison of measurements between different machines. Finally, the results presented herein represent the first step of a validation strategy of the loadcapacity indices, as further analyses are needed to evaluate the association of the indices with disease risk as well as to discern the superiority of a specific load-capacity model for the classification of body composition phenotypes and disease-risk prediction. These analyses are warranted to evaluate the discriminatory accuracy of the proposed models.

\section{Conclusions}

SO has emerged as a potential diagnostic model integrating the effects of low muscle mass and high adiposity on physiological functions. However, a unanimous agreement on the operationalisation of $\mathrm{SO}$ diagnosis is lacking, which is reflected in the heterogeneous association between SO and disease risk. The current study has developed an integrated diagnostic approach for the definition of body composition phenotypes based on a load-capacity model (FM:FFM and TrFM:ASM), which may be particularly useful for the identification of $\mathrm{SO}$ cases. The accuracy of the proposed diagnostic models for the prediction of disease risk remains to be tested in future studies.

\section{Acknowledgements}

Acknowledgements: The authors would like to thank Dr Thomas Kelly for his help with the development of the 
reference curves. Financial support: This research received no specific grant from any funding agency in the public, commercial or not-for-profit sectors. Conflict of interest: None. Authorship: M.S., C.M.P. and P.T.K. conceptualised and designed the project, drafted the article, critically revised it and approved the final version. E.M. conducted the statistical analyses, critically revised the manuscript and approved the final version. J.C.K.W., S.B. and S.H. critically revised the manuscript and approved the final version. Ethics of human subject participation: Approval for NHANES 1999-2004 was obtained from the National Center for Health Statistics Institutional Review Board (Protocol \#98-12).

\section{Supplementary material}

To view supplementary material for this article, please visit http://dx.doi.org/10.1017/S1368980014001918

\section{References}

1. Prentice AM \& Jebb SA (2001) Beyond body mass index. Obes Rev 2, 141-147.

2. Ness-Abramof R \& Apovian CM (2008) Waist circumference measurement in clinical practice. Nutr Clin Pract 23, 397-404.

3. Bosy-Westphal A, Geisler C, Onur S et al. (2005) Value of body fat mass vs anthropometric obesity indices in the assessment of metabolic risk factors. Int J Obes (Lond) $\mathbf{3 0}$, 475-483.

4. Janssen I, Katzmarzyk PT \& Ross R (2002) Body mass index, waist circumference, and health risk: evidence in support of current National Institutes of Health guidelines. Arch Intern Med 162, 2074-2079.

5. Ashwell M, Gunn P \& Gibson S (2012) Waist-to-height ratio is a better screening tool than waist circumference and BMI for adult cardiometabolic risk factors: systematic review and meta-analysis. Obes Rev 13, 275-286.

6. Woo J, Ho SC, Yu AL et al. (2002) Is waist circumference a useful measure in predicting health outcomes in the elderly? Int J Obes Relat Metab Disord 26, 1349-1355.

7. Chang S-H, Beason TS, Hunleth JM et al. (2012) A systematic review of body fat distribution and mortality in older people. Maturitas 72, 175-191.

8. Müller MJ, Lagerpusch M, Enderle J et al. (2012) Beyond the body mass index: tracking body composition in the pathogenesis of obesity and the metabolic syndrome. Obes Rev 13, 6-13.

9. Thibault R, Genton L \& Pichard C (2012) Body composition: why, when and for who? Clin Nutr 31, 435-447.

10. Prado CM, Wells JC, Smith SR et al. (2012) Sarcopenic obesity: a critical appraisal of the current evidence. Clin Nutr 31, 583-601.

11. Baumgartner RN (2000) Body composition in healthy aging. Ann N Y Acad Sci 904, 437-448.

12. Roubenoff R (2004) Sarcopenic obesity: the confluence of two epidemics. Obes Res 12, 887-888.

13. Zamboni M, Mazzali G, Fantin F et al. (2008) Sarcopenic obesity: a new category of obesity in the elderly. Nutr Metab Cardiovasc Dis 18, 388-395.

14. Waters DL \& Baumgartner RN (2011) Sarcopenia and obesity. Clin Geriatr Med 27, 401-421.

15. Batsis JA, Barre LK, Mackenzie TA et al. (2013) Variation in the prevalence of sarcopenia and sarcopenic obesity in older adults associated with different research definitions: dual-energy X-ray absorptiometry data from the National
Health and Nutrition Examination Survey 1999-2004. J Am Geriatr Soc 61, 974-980.

16. Schautz B, Later W, Heller M et al. (2012) Total and regional relationship between lean and fat mass with increasing adiposity - impact for the diagnosis of sarcopenic obesity. Eur J Clin Nutr 66, 1356-1361.

17. Jackson AS, Janssen I, Sui X et al. (2012) Longitudinal changes in body composition associated with healthy ageing: men, aged 20-96 years. Br J Nutr 107, 1085-1091.

18. Kelley DE, Slasky BS \& Janosky J (1991) Skeletal muscle density: effects of obesity and non-insulin-dependent diabetes mellitus. Am J Clin Nutr 54, 509-515.

19. Kent-Braun JA, Ng AV \& Young K (2000) Skeletal muscle contractile and noncontractile components in young and older women and men. J Appl Physiol 88, 662-668.

20. Wells JCK (2009) Historical cohort studies and the early origins of disease hypothesis: making sense of the evidence. Proc Nutr Soc 68, 179-188.

21. Wells JC (2011) The thrifty phenotype: an adaptation in growth or metabolism? Am J Hum Biol 23, 65-75.

22. Booth FW, Roberts CK \& Laye MJ (2012) Lack of exercise is a major cause of chronic diseases. Compr Physiol 2, $1143-1211$.

23. Bremer AA \& Jialal I (2013) Adipose tissue dysfunction in nascent metabolic syndrome. $J$ Obes $\mathbf{2 0 1 3}, 8$.

24. Siervo M \& Jebb SA (2010) Body composition assessment: theory into practice: introduction of multicompartment models. IEEE Eng Med Biol Mag 29, 48-59.

25. Andreoli A, Scalzo G, Masala S et al. (2009) Body composition assessment by dual-energy X-ray absorptiometry (DXA). Radiol Med 114, 286-300.

26. Grundy SM (2004) Obesity, metabolic syndrome, and cardiovascular disease. J Clin Endocrinol Metab 89, 2595-2600.

27. Centers for Disease Control and Prevention (2012) National Health and Nutrition Examination Survey. http://www.cdc. gov/nchs/nhanes/irba98.htm (accessed September 2013).

28. Kelly TL, Wilson KE \& Heymsfield SB (2009) Dual energy X-ray absorptiometry body composition reference values from NHANES. PLoS One $\mathbf{4}$, e7038.

29. Centers for Disease Control and Prevention (2000) National Health and Nutrition Examination Survey: body composition procedures manual. http://www.cdc.gov/nchs/data/ nhanes/BC.pdf (accessed September 2013).

30. Centers for Disease Control and Prevention (2008) National Health and Nutrition Examination Survey: technical documentation for the 1999-2004 dual energy X-ray absorptiometry (DXA) multiple imputation data files. http://www. cdc.gov/nchs/data/nhanes/dxa/dxa_techdoc.pdf (accessed September 2013).

31. Pan H \& Cole TJ (2004) A comparison of goodness of fit tests for age-related reference ranges. Stat Med 23, 1749-1765.

32. Siervo M, Stephan BCM, Nasti G et al. (2012) Ageing, adiposity indexes and low muscle mass in a clinical sample of overweight and obese women. Obes Res Clin Pract 6 , e63-e70.

33. Bertoli S, Battezzati A, Testolin G et al. (2007) Evaluation of air-displacement plethysmography and bioelectrical impedance analysis vs dual-energy X-ray absorptiometry for the assessment of fat-free mass in elderly subjects. Eur J Clin Nutr 62, 1282-1286.

34. Bosy-Westphal A, Mast M, Eichhorn C et al. (2003) Validation of air-displacement plethysmography for estimation of body fat mass in healthy elderly subjects. Eur J Nutr 42, 207-216.

35. Bedogni G, Agosti F, De Col A et al. (2013) Comparison of dual-energy X-ray absorptiometry, air displacement plethysmography and bioelectrical impedance analysis for the assessment of body composition in morbidly obese women. Eur J Clin Nutr 67, 1129-1132. 
36. Grijalva-Eternod CS, Lawlor DA \& Wells JCK (2013) Testing a capacity-load model for hypertension: disentangling early and late growth effects on childhood blood pressure in a prospective birth cohort. PLoS One 8, e56078.

37. Sternfeld B, Ngo L, Satariano WA et al. (2002) Associations of body composition with physical performance and selfreported functional limitation in elderly men and women. Am J Epidemiol 156, 110-121.

38. Lim KI, Yang SJ, Kim TN et al. (2010) The association between the ratio of visceral fat to thigh muscle area and metabolic syndrome: the Korean Sarcopenic Obesity Study (KSOS). Clin Endocrinol (Oxf) 73, 588-594.

39. Auyeung T, Lee J, Leung J et al. (2013) Adiposity to muscle ratio predicts incident physical limitation in a cohort of 3,153 older adults - an alternative measurement of sarcopenia and sarcopenic obesity. Age (Dordr) 35, 1377-1385.

40. Schoeller DA, Tylavsky FA, Baer DJ et al. (2005) QDR 4500A dual-energy X-ray absorptiometer underestimates fat mass in comparison with criterion methods in adults. Am J Clin Nutr 81, 1018-1025. 